\title{
Half-Sweep Imaging for Depth from Defocus
}

\author{
Shuhei Matsui, Hajime Nagahara, and Rin-ichiro Taniguchi \\ Graduate School of Information Science and Electrical Engineering, Kyushu University. \\ 744 Motooka, Nishi-ku, Fukuoka 819-0395, Japan \\ \{matsui, nagahara, rin\}@limu.ait.kyushu-u.ac.jp
}

\begin{abstract}
Depth from defocus (DFD) is a technique to recover the scene depth from defocusing in images. DFD usually involves two differently focused images (near-focused and far-focused) and calculates the size of the depth blur in the captured images. In recent years, the coded aperture technique, which uses a special pattern for the aperture to engineer the point spread function (PSF), has been used to improve the accuracy of DFD estimation. However, coded aperture sacrifices an incident light and loses a SNR of captured images which is needed for the accurate estimation. In this paper, we propose a new computational imaging, called half-sweep imaging. Half-sweep imaging engineers PSFs for improving DFD and maintaining the SNR of captured images. We confirmed the advantage of the imaging in comparison with conventional DFD and coded aperture in experiments.
\end{abstract}

Keywords: computational photography, depth from defocus, image deblurring.

\section{Introduction}

There are many methods, referred to as depth from defocus (DFD) techniques [9], [11], for estimating scene depths using a single camera. The methods use depth blurs (i.e., blurring that depends on the scene depth) that appear in captured images. DFD usually employs a pair of images one being near-focused and the other being far-focused to determine differences in sizes of depth blurs resulting from depth differences in a scene. However, the circular shape of the aperture of a regular camera is not beneficial for DFD estimation, since the aperture moderately affects depth blurring. For more robust DFD estimation, many researchers have investigated coded aperture techniques [3], [5], [13]. Such techniques use special patterns for the camera aperture to control the shape of the point spread function (PSF). Additionally, it is well known that the shape of the PSF directly affects the frequency response of an imaging system, which is described by the optical transfer function in the field of optics. We can select aperture patterns that drastically change the PSF shape in the image domain or its frequency response in the Fourier domain according to scale changes of the PSF due to object depth differences, thus achieving more accurate DFD estimation in discriminating scene depths. However, the use of a coded aperture attenuates the intensity of captured images, since incident light from the scene is blocked in engineering the PSFs. The attenuation decreases the signal-to-noise ratio (SNR) of the images and limits the improvement of DFD estimation. 
In this paper, we propose a new imaging operation called half-sweep imaging for DFD estimation. DFD has sometimes ignored the quality of the recovering image. We focus to realize high quality of all-in-focus image reconstruction as well as robust DFD estimation for considering to visualization in computational photography. The technique is inspired by focus sweeping [7], [4] and is extended to DFD applications. Halfsweep imaging obtains two images by sweeping the focus during the image exposure time. It has the advantage of a higher SNR for captured images, since we can engineer the image PSFs even if a camera aperture is open. The operation requires the continuous changing of the lens focus or sweeping of an image sensor, which is easy to implement since we can utilize an auto-focusing mechanism or an actuator for image stabilization that current commercial cameras already possess. Moreover, the method has complete compatibility with regular imaging and adaptivity to scene depth when we stop the sweeping motion or freely adjust the sweeping length and positions. Employing the proposed method, we integrate multiple PSFs with different focus settings obtained by focal sweeping to control the frequency responses of imaging PSFs. We split a sweep into half regions to capture images. The two obtained images are captured for the same scene, but using different PSFs (i.e., transfer functions of imaging). As a result, one of the PSFs and captured images has zero-crossing in its frequency response, which helps with depth estimation, and the sum of PSFs has a broadband spectrum, which allows recovery of a better all-in-focus image.

\section{Related Work}

Many researchers have proposed PSF engineering methods to improve DFD estimation. As an early work on coded apertures, Hiura and Matsuyama [3] used three or four pin holes as the aperture of a multiple-focus camera. They used three differently focused images captured by the camera and realized robust depth estimation. However, this aperture coding was far from optimal.

Levin et al. [5] proposed using an aperture with a pattern more distinguishable than that of a conventional circular aperture. They defined K-L divergence as a metric of the PSF scale difference due to depth difference and found an optimal pattern for DFD estimation by maximizing the metric. The Fourier spectrum of the pattern contains many zero-crossings and their positions are displaced when the blur size changes owing to the depth difference. If we use a different size of the PSF for deconvolution, the recovered image has severe artifacts from the disagreement with the true PSF spectrum. The artifacts increase the penalty for misrecognizing the depth and improve the stability of DFD estimation. As a result, they allow DFD estimation from a single image, while common DFD methods require at least two differently focused images to solve ambiguity in the blurred image due to texture. However, the aperture is not suited to recovering an all-in-focus image through deconvolution, since the frequency response of a zero-crossing point is such that we have zero information at that frequency.

Zhou et al. [13] proposed a coded aperture pair to recover a high-quality focused image and estimate depth. It is well known that a broadband PSF in the Fourier domain is favorable for blurred-image recovery through deconvolution, since it provides image information through the entire frequency range even though the captured image 
is blurred [12], [14]. However, as mentioned for Levin et al.'s work [5], zero-crossings are favorable for depth estimation. These properties are not compatible with each other when only using a single aperture pattern. There is a dilemma in practical DFD applications that it is necessary to recover the true texture for accurate depth estimation, but recovering the texture requires knowledge of the correct depth information. Therefore, Zhou et al. [13] proposed the use of a pair of coded apertures that optimize image reconstruction and depth estimation simultaneously. In the case of their proposed aperture pair, the frequency response of a single PSF has zero-crossings, but the sum of PSFs has a broadband since the PSFs have complementary responses. The need to replace two lenses with the coded aperture pair remains a difficult problem in image capturing.

A programmable-aperture camera that can quickly switch aperture patterns has been developed [8]. Green et al. [2] proposed a multiple-aperture camera that uses special mode mirrors. There are examples of implementations that have realized easy capturing and increasing flexibility for multiple coded apertures. However, PSF engineering using a coded aperture has an intuitive problem that the SNR of the image is lower than that of the conventional DFD measurement, since the aperture blocks incident light in controlling the PSF shape. Therefore, there is the limitation that noise in the image destabilizes depth estimation and contaminates the recovered image.

Wavefront coding engineers the PSF without blocking incident light unlike the case for a coded aperture. Employing this method, a special optical element called a phase plate is placed at the position of the camera aperture. The phase plate controls the wavefront of rays according to the positions in aperture open. Dowski et al. [1] proposed a phase plate for DFD estimation whose PSF spectrum has many zero-crossings. Levin et al. [6] theoretically analyzed the upper bound of the PSF response for image deblurring and designed optics called a lattice focus lens to realize the PSF. The lens can be used to estimate the scene depth and achieve optimal defocus deblurring, since the PSF of the lattice focus lens is depth-variant. Wavefront coding engineers the PSF with an open aperture and realizes image acquisition with a higher SNR. However, the cost of the phase plate is expensive and its property is not adaptive to a scene.

Nagahara et al. [7], [4] proposed focus sweep imaging that moves focus points during the image integration time to capture a single image. This method integrates different scales of PSFs to realize PSFs that have broadband frequency response and invariant shapes through the entire scene depth. They proposed applying this imaging operation to an extended depth of field by deblurring without any depth estimation or knowledge. The advantages of the focal sweep are a higher SNR of captured image, compatibility of regular photograph and flexibility for scene. Hasinoff et al. [10] discussed the optimal number of focal stack images across a scene depth for various imaging systems. They applied focal sweep imaging to acquire focal stack (multiple) images to obtain a best allin-focused image. They showed it in simulation and did not compaired DFD accuracy in the paper.

\section{Half-Sweep Imaging}

Focus sweep imaging [7], [4] sweeps the focal plane through a scene during the image exposure time. It is achieved by moving the lens or image sensor position along the optical axis. We can manipulate the PSF by controlling the range or speed of the 


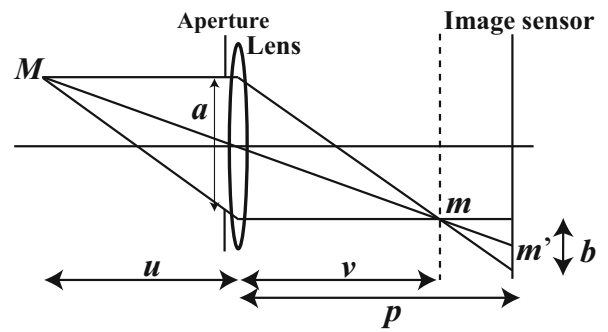

Fig. 1. Projective geometry of lens

sweeping. In this paper, we propose an extension of focal sweep imaging called halfsweep imaging for DFD application. Full-sweep imaging [7], [4] sweeps the focal plane through the entire depth of the target scene in the exposure time to realize the extended depth of field. Our half-sweep imaging splits the sweep range into two regions and captures two images corresponding to the front half and back half of the sweeping regions. Consequently, we capture two images that have depth-variant blurs (PSFs) for DFD estimation, while the original full sweep obtains depth-invariant blurs for deblurring. In this section, we present the properties and advantages of PSFs in half-sweep imaging.

Figure 11 shows the projective geometry where the image sensor is at a distance $p$ from a lens with focal length $f$, and the aperture diameter is $a$. Incident rays from a scene point $M$ at the distance $u$ converge to the focused point $m$ at a distance $v$ from the lens. The relation between $u$ and $v$ is described by the Gaussian lens law:

$$
\frac{1}{f}=\frac{1}{u}+\frac{1}{v} .
$$

As shown in the figure, if an image sensor is placed at a distance $p$ from the lens, $M$ is imaged to $m^{\prime}$ with blur on the sensor. The diameter of the blurred circle $b$ is given by

$$
b(p)=\frac{a}{v}|(v-p)| .
$$

The PSF is a function of the distribution of light energy within the blurred circle. We consider here $r$ to be the distance of an image point from the center $m^{\prime}$ of the blurred circle, and the PSF is denoted $P(r, u, p)$. The PSF is often modeled as a pillbox function:

$$
P(r, u, p)=\frac{4}{\pi b^{2}} \prod\left(\frac{r}{b}\right),
$$

where $\prod(x)$ is the rectangle function, which has a value 1 if $|x|<1 / 2$ and 0 otherwise. This is the PSF function of an object placed at $u$ when the sensor position is fixed at $p$ as in regular imaging with a common camera.

In half-sweep imaging, the sensor moves from $p_{0}$ to $p_{2}$ along the optical axis of the camera as shown in Figure 2-a. We assume that focus points of all objects in a scene lie between $p_{0}$ and $p_{2}$. The half-sweep imaging captures two images $f_{1}$ and $f_{2}$ with exposures $e_{1}$ and $e_{2}$ as shown in Figure2 2-b. The sensor motion is modeled as a function 


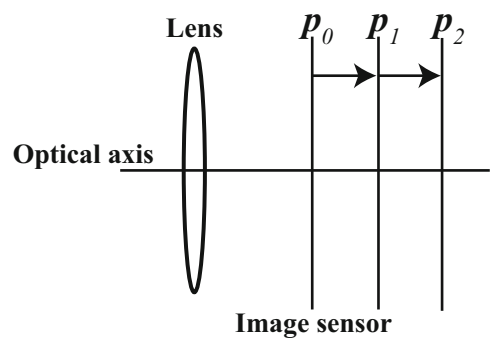

a. Sensor motion

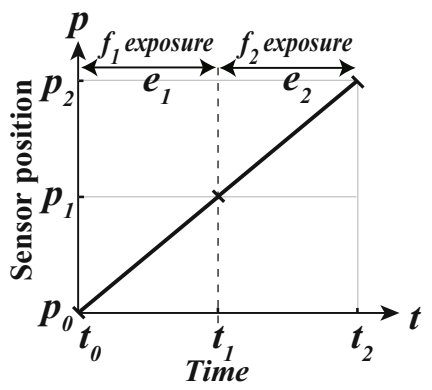

b. Motion and image integrations

Fig. 2. Half sweep imaging

of time $p(t)=s t+p_{0}$ if the sensor moves with constant speed $s$. The relation between the sensor motion and exposure time is shown in Figure 2 -b. This figure describes that the exposures $e_{1}$ and $e_{2}$ for capturing the images $f_{1}$ and $f_{2}$ correspond to sweep regions from $p_{0}$ to $p_{1}$ and from $p_{1}$ to $p_{2}$ respectively. Hence, we obtain two images with different integrations of different blurred images focusing at positions between $p_{0}$ and $p_{1}$ or $p_{1}$ and $p_{2}$. It is easy to realize half sweeping by simply changing the shutter timings and the exposure time from those for full sweeping. An imaging process can be modeled by convolution of the PSF function:

$$
f_{i}=h_{i} \otimes f_{0}+\xi, \quad i=1,2,
$$

where $f_{i}$ is the observed image, $h_{i}$ is the half-sweep PSF, $f_{0}$ is the latent in-focus image and $\xi$ is the image noise, which is assumed to be Gaussian white noise $N\left(0, \sigma^{2}\right)$. Normally, the shape of a PSF is determined by the aperture size and object depth as described by Equation 3. Meanwhile, the half-sweep PSF $h_{i}$ is modeled by integration of PSFs at multiple sensor positions $p$ through the sweeping regions during the exposure time. This is described by

$$
h_{i}(r, u)=\int_{p_{i-1}}^{p_{i}} P(r, u, p) d p, \quad i=1,2,
$$

where $p_{i}(i=0,1,2)$ is the position of the image sensor. The sensor moves from $p_{i-1}$ to $p_{i}$ during exposure time $e_{i}$. If we assume that the integrated blur model is a pillbox function as described in Equation 3 the half-sweep PSF is modeled by

$$
h_{i}(r, u)=\frac{u f}{(u-f) \pi a s p_{i}}\left(\frac{\lambda_{p_{i-1}}+\lambda_{p_{i}}}{r}-\frac{2 \lambda_{p_{i-1}}}{b\left(p_{i-1}\right)}-\frac{2 \lambda_{p_{i}}}{b\left(p_{i}\right)}\right), i=1,2,
$$

where $b(p)$ is the diameter of the blurred circle at position $p$, and $\lambda_{p}=1$ if $b(p) \geq 2 r$ and 0 otherwise.

Figure 3-a shows simulated half-sweep PSFs $h_{1}, h_{2}$ modeled by Equation 6 and their average PSF $h_{\text {all }}$ at four different scene depths. The four different depth positions $u$ are decided by the relation of the lens law so that the corresponding focal position $v$ is at constant intervals on the sensor side. The average PSF $h_{\text {all }}$ is simply derived according 


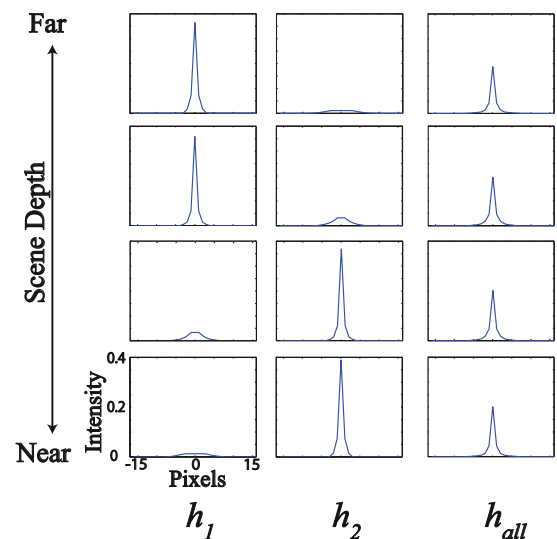

a. PSF profile

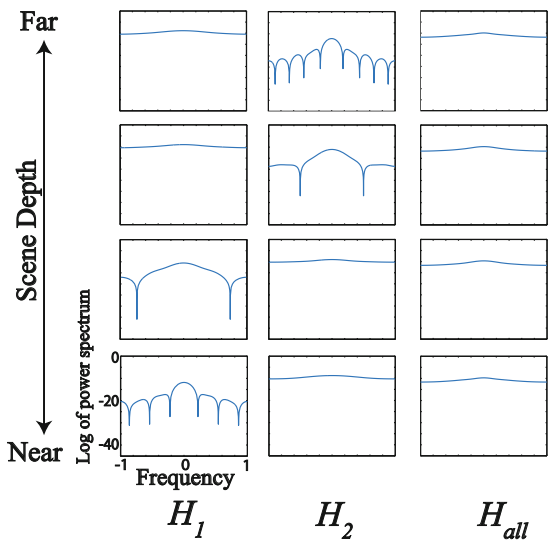

b.FFT profile

Fig. 3. Half sweep PSF

to $h_{\text {all }}=\left(h_{1}+h_{2}\right) / 2$. Therefore, we consider $h_{\text {all }}$ to be the same as the full sweep PSF [7], [4], since the sum of $h_{1}$ and $h_{2}$ is an integration of different focal images through the entire swept region. The figure shows that PSF shapes $h_{1}$ and $h_{2}$ change according to the depth difference, while shape of the average PSF $h_{\text {all }}$ does not vary visually. Figure 3-b shows the logarithms of power spectrums of these three PSFs with different scene depths in the frequency domain, where $H_{1}, H_{2}$ and $H_{\text {all }}$ are the discrete Fourier transforms of $h_{1}, h_{2}$ and $h_{\text {all }}$ respectively. This plot corresponds to Figure 3 -a. The spectrums of $H_{1}$ and $H_{2}$ change according to depth. We also see zero-crossings in one of the spectrums. On the other hand, $H_{\text {all }}$ has a broadband spectrum. Levin et al. [5] and Zhou et al. [13] claimed that PSFs having zero-crossings are a useful property of the DFD measurement and improve depth discrimination. Additionally, it is well known that broadband PSFs are beneficial for defocus deblurring [12], [14], [7], [4], [13] and allow the generation of good quality all-in-focus images in DFD application.

\section{DFD Algorithm for Half-Sweep Imaging}

In this section, we propose a method for estimating a depth map and an all-in-focus image from two images captured by half-sweep imaging. Half-sweep imaging is expressed as Equation 4 This can be written in the Fourier domain as

$$
F_{i}^{(d)}=F_{0} \cdot H_{i}^{(d)}+N, \quad i=1,2,
$$

where $F_{i}^{(d)}$ is the Fourier transform of a captured images $(i=1,2)$ at depth $d, F_{0}$ is the Fourier transform of a latent all-in-focus image, $H_{i}^{(d)}(i=1,2)$ is the Fourier transform of a half-sweep PSFs at depth $d$ and $N$ is the Fourier transform of noise. We consider here the problem in which we estimate the all-in-focus image $F_{0}$ and unknown scene 
depth $d$ from Equation 7 . Generally, the image $F_{0}$ is given by deconvolution. We use the Wiener deconvolution:

$$
\hat{F}_{0}=\frac{F \cdot \bar{H}}{\left|H^{2}\right|+|C|^{2}},
$$

where $\bar{H}$ is a complex conjugate of $H$ and $|H|^{2}=H \cdot \bar{H} \cdot C$ represents $\sigma / A^{\frac{1}{2}}$, where $A$ is defined over the power distribution of natural images according to the $1 / f$ law. The original Wiener deconvolution was designed to deblur one blurred image, and we propose to use the extended method in our half-sweep imaging. As shown in section 3 $h_{\text {all }}$, which is the average of $h_{1}$ and $h_{2}$ kernels, has a broadband frequency response for each depth. Additionally, $f_{\text {all }}$, which is the average image of $f_{1}$ and $f_{2}$, has broadband image information, since we can assume that the image $f_{\text {all }}$ is captured by the $h_{\text {all }}$ kernel. The property of addition is maintained over the Fourier transform. Hence, we obtain the Fourier transforms of the average kernel and the image as

$$
F_{\text {all }}=\frac{F_{1}+F_{2}}{2}, \quad \overline{H_{\text {all }}^{(d)}}=\frac{\overline{H_{1}^{(d)}}+\overline{H_{2}^{(d)}}}{2} .
$$

We can extend Wiener deconvolution to half-sweep imaging by substituting Equation 9 into Equation 8 .

$$
{\hat{F_{0}}}^{(d)}=\frac{\left(F_{1}+F_{2}\right) \overline{\left(H_{1}^{(d)}+H_{2}^{(d)}\right)}}{\left|H_{1}^{(d)}+H_{2}^{(d)}\right|^{2}+4|C|^{2}} .
$$

We consider that the error between observed images and estimated observed images must be minimum when the estimated depth $d$ is correct. Therefore, we defined a cost function to estimate depth $d$ is expressed as

$$
W^{(d)}=\sum_{i=1,2}\left|\operatorname{IFFT}\left(\hat{F}_{0}^{(d)} \cdot H_{i}^{(d)}-F_{i}\right)\right|,
$$

where IFFT is the 2D inverse Fourier transform and $\hat{F}_{0}$ is derived from Equation 10 The cost function $W^{(d)}$ represents the error between the reconstructed images and the captured images; therefore, $W^{(d)}$ is a measure of how close $d$ is to the actual scene depth $d^{*}$. We estimate depths to find the minimum $W^{(d)}$ for each pixel $(x, y)$ using

$$
U(x, y)=\arg \min _{d \in D} W^{(d)}(x, y) .
$$

We also obtain an all-in-focus image $I$ from the estimated depth map $U$ as

$$
I(x, y)=\hat{F}_{0}^{(U(x, y))}(x, y)
$$

\section{Performances Analysis}

We carried out simulation experiments to evaluate the performance of our half-sweep imaging. In this section, we denote the object position as $u$, the focal point $v$ and sensor position $p$ as shown in Figure 1. We assumed that the scene is a synthetic staircase 
Table 1. Correspondence among step number, object depth and focus position ( $f=9 \mathrm{~mm}$ )

\begin{tabular}{l|ccccccccc}
\hline Depth step & Step1 & Step5 & Step10 & Step15 & Step20 & Step25 & Step30 & Step35 & Step40 \\
\hline Object: $u[\mathrm{~mm}]$ & 2034 & 321 & 160 & 109 & 83.6 & 68.6 & 58.5 & 51.4 & 46.1 \\
\hline Focus: $v[\mathrm{~mm}]$ & 9.040 & 9.260 & 9.535 & 9.810 & 10.085 & 10.360 & 10.64 & 10.91 & 11.185 \\
\hline
\end{tabular}

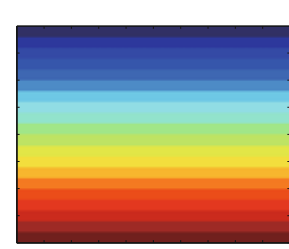

a. Ground Truth

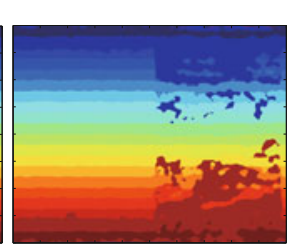

b. Conventional DFD

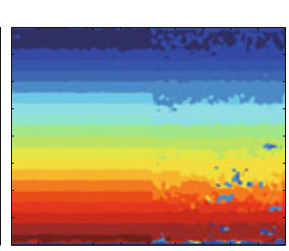

c. Coded aperture pair

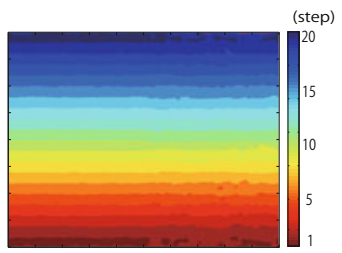

d. Half-sweep imaging

Fig. 4. Estimated depth map

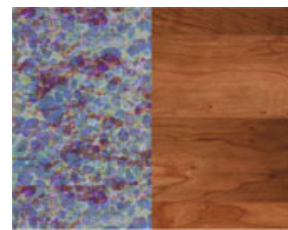

a. True texture

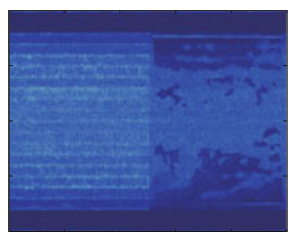

b. Conventional DFD

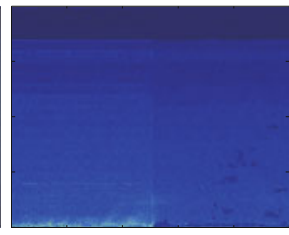

c. Coded aperture pair

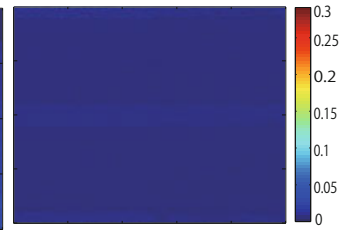

d. Half-sweep imaging

Fig. 5. Error map of deblurred image

scene as shown in Figure 4-a. These depth maps have false-color representation with red indicating locations far (step 1) from the camera and blue locations near (step 20) the camera. The scene has two textures, one with strong and dense patterns and the other of natural wood with weak texture as shown in Figure 5 -a. The physical object depths $u$ are from 2034 to $83.6 \mathrm{~mm}$ from a camera lens. The corresponding focal point $v$ varies from 9.04 to $10.085 \mathrm{~mm}$ behind the lens according to the lens law of Equation 1 We divided the possible range of the focal position $v$ into 20 uniform steps ( $\Delta v=0.055$ $\mathrm{mm}$ ) so that the depth blur must change by a similar ratio ( 0.5 pixels for each step) in an image. Table 1 gives the conversion among a step number, the corresponded object depth $u$ and the focal position $v$ for easy understanding of the relations. The focal length and F-number of a lens are taken as $f=9 \mathrm{~mm}$ and $f / 1.4$ in our setting. Under these settings, we simulated captured images through convolution with theoretical PSFs modeled by the pillbox function described by Equation 6 We set integration intervals to half by half of the target depth range for half-sweep DFD. When the depth range is 20 steps in this case, the intervals are 1 to 10 and 11 to 20 steps. The corresponding sensor positions are $p_{0}=9.04 \mathrm{~mm}, p_{1}=9.945 \mathrm{~mm}$ and $p_{2}=10.085 \mathrm{~mm}$ for equation 5 . The conventional DFD used far-focused (step 1, $p=9.04 \mathrm{~mm}$ ) and near-focused (step 20, $p$ $=10.085 \mathrm{~mm}$ ) images with an open circular aperture. The coded aperture used two farfocused (step 20, $p=9.04 \mathrm{~mm}$ ) images captured by an aperture pair [13](i.e., aperture difference is the depth key). We estimated scene depth maps and all-in-focus images using the proposed DFD algorithm as mentioned in Section 4 . For the conventional and coded-aperture DFD estimation, we used Zhou's DFD algorithm [13] for comparison. 


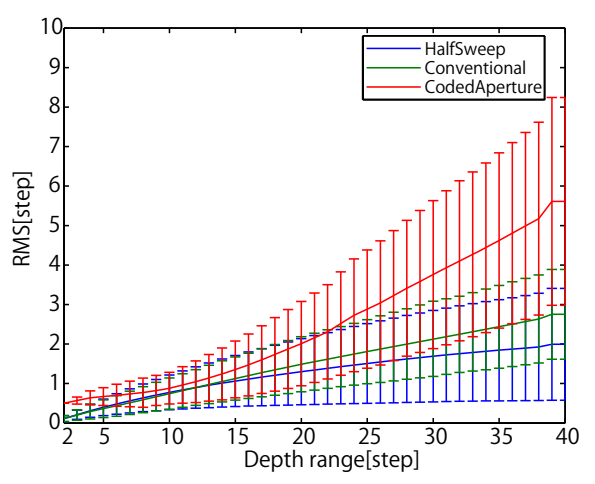

a. RMS vs Depth range

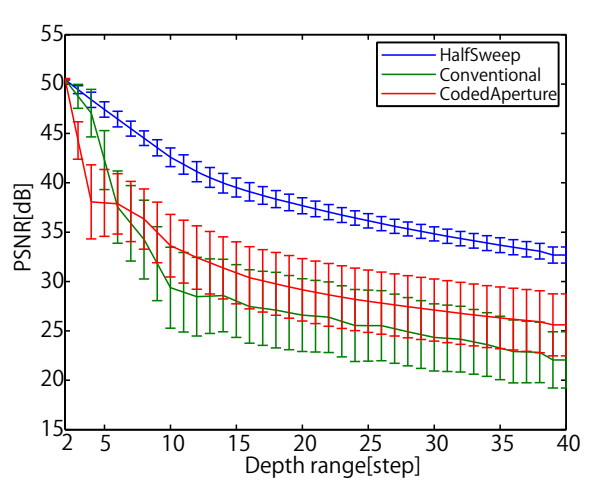

b.PSNR vs Depth range

Fig. 6. Simulation results

Figure 4 shows the depth estimation results. The figure shows the depth maps of (a) the ground truth, (b) the conventional DFD method, (c) Zhou's coded aperture pair and (d) our proposed method by capturing half-sweep imaging. We see that the strong texture on the left side of the scene does not differ greatly among the methods. However, there are large differences for the weak texture on the right side, with our proposed halfsweep imaging having the best performance. Figure 4-b, the result for conventional DFD estimation, shows large error around the central depth, and Figure 4tc, the result for coded-aperture-pair DFD estimation, shows error for the entire depth range. On the other hand, Figure 4 -d, the result for our method, shows greater robustness, although the scene has weak texture. Figures 5-b, c, d show difference images between the estimated images and the true texture as shown in Figure 5-a, since it is difficult to recognize the error in the estimated images. The figures are shown by false color representation and the color bar indicates the errors in normalized intensity (i.e., maximum intensity is 1.0). Figure 5tb shows large reconstruction errors for the center of the image, since captured images have large blurs and high-frequency information was lost in the center of the image in conventional DFD estimation. Figure 5-c shows that the recovery errors increase where the object depth approaches a far position. It is difficult to distinguish the difference between the coded aperture pair where the size of blur is small or in focus, since the method of the coded aperture pair employs the shape difference between the apertures as a depth key. Figure 5-d shows that the proposed recovery method produces errors that are smaller and more uniform.

We also compared numerical qualities among these methods. In this experiment, we used similar setting to Figure 4, but we changed the object depth range (the number of stairs) from 2 to 40 steps. Table 1 also shows the conversion of the object depth. We used arbitrary 30 images downloaded from flickr as scene textures for generating simulated images. Figure 6 $\mathrm{a}$ a and $\mathrm{b}$ show the root-mean-square (RMS) of the estimated depth errors and the peak signal-to-noise ratios (PSNR) of the recovered images against the object ranges. In these figures, line plots indicate the average values of the RMS 


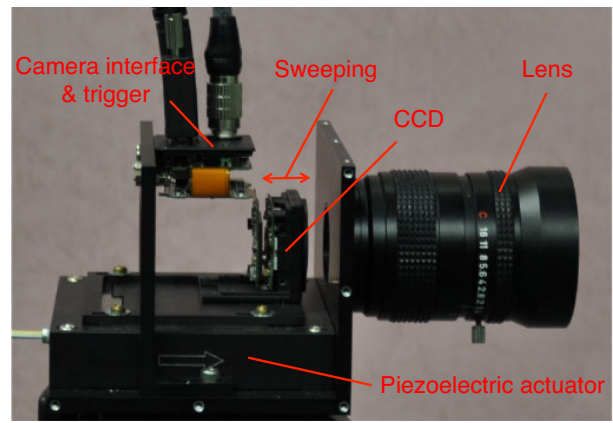

Fig. 7. Prototype focal sweep camera

error or PSNR, and the error bars indicate the standard deviations of 30 variations of the textures. The standard deviations imply that the each result is deviated depending on the texture difference. Figure 6-a shows that all of the methods are getting worse if the depth range are enlarged, since bigger size of blur must be used for estimating larger depth range and it is difficult to estimate the blur size when the size is larger. Half sweep DFD is still better performance for estimating the depth than the others. Figure 6-b also shows the similar results that PSNR is getting worse when the depth range is enlarged among the all methods. Yet, it is obvious that the PSNR of the proposed method is far better than that of the others. We can also see that the standard deviation of the proposed method is smaller than the others. It means that half sweep DFD is more robust to recover the images independent to the scene texture variety. These figures show that the proposed method outperforms both depth estimation and the recovered image quality.

We confirm that the proposed method of half-sweep imaging has the best performance in terms of estimating the scene depth and recovering an all-in-focus image. This is due to one of the proposed half-sweep imaging PSFs having zero-crossings and their sum having a broadband spectrum in the Fourier domain.

\section{Real Implementation and Experiments}

We evaluated our half-sweep imaging for real images captured by a prototype camera. Figure 7 shows the prototype camera for realizing half-sweep imaging. The camera consists of a 1/3" Sony CCD (with $1032 \times 776$ pixels) mounted on a Physik Instrument $\mathrm{P}-628.1 \mathrm{CL}$ translation stage. This stage is driven by a piezoelectric actuator and the range of translation is 800 microns. We attached a Tokina $12.5 \mathrm{~mm}$ lens and the Fnumber was set to $f / 1.4$ in this experiment. The shutter of the CCD and the actuator were controlled with by signals generated by PC. They were completely synchronized for realizing half-sweep imaging.

A target scene that we captured in this experiment is shown in Figures 8 and 9 . There are four objects at different depths in front of a wall in the scene. The range of scene 
depth was 340 to $750 \mathrm{~mm}$ from the camera lens. The actuator needed to translate 225 microns from start to end positions to covering the entire scene depth. Figure 9 - a shows the images captured using the prototype camera with half-sweep imaging. The images $f_{1}$ and $f_{2}$ were captured by the front half and back half of the sensor sweeping. Figures 8 -a show images again captured using the prototype camera but with near-focus and far-focus positions (not sweeping) for conventional DFD. We captured measured PSFs for both half-sweep imaging and conventional DFD estimation at ten depths using a point light source before the experiments.

We estimated the object depths and recovered all-in-focus images using the input images and measured PSFs. Figures 9.b, c show the results of the recovered all-in-focus images and the depth map of the scene. We employed the proposed DFD method as mentioned in Section 4 Figures 8 $\mathrm{b}$, $\mathrm{c}$ show the results of conventional DFD estimation for comparison. Comparing Figures 9-d and 8-d, we see that both depth maps show the depth differences among the objects and we cannot see a strong advantage for one method over the other. It was caused by that the scene has relatively strong texture unlike the scene in the simulation. Hence, the difference was not appeared between the methods.

Comparing Figures 9 b and 8 b, we see that the recovered focused images have large differences. The image obtained through conventional DFD estimation has many artifacts such as enhanced noise and ringging artifacts. We also see that some portion of the texture is still blurred even after deconvolution because of depth estimation errors. Figures 10 clearly show the differences for magnified portions of the images. Figure 10 . c shows the ground truth textures captured for the same scene with a small aperture setting of $f / 16$. The proposed method does not provide an image that is identical to the ground truth but has far better performance than the conventional DFD approach. The experiments confirm that our proposed method has an advantage over the conventional DFD and works in a real implementation.

\section{Conclusion}

This paper proposes a new computational imaging technique called half-sweep imaging and a processing method for DFD estimation. The sensor sweeps during the exposure time to capture two images. We show that PSFs of the proposed half-sweep imaging simultaneously have zero-crossings and broadband properties in the Fourier domain. We realized robust depth estimation and high-quality image recovery from the contribution of the PSF properties. We confirmed the advantage of our half-sweep imaging over previous methods in simulation and real experiments. We implemented a prototype camera that incorporates a piezoelectric actuator to sweep an image sensor in real experiments. However, the sweeping operation can be more easily implemented for commercial cameras; e.g., utilizing the auto-focusing mechanism. Half-sweep imaging has the advantages of obtaining a higher SNR for images, having flexibility such that it can adapt to the scene depth, and having complete compatible with regular imaging. Hence, it is applicable for a wide range of products such as digital still cameras. 


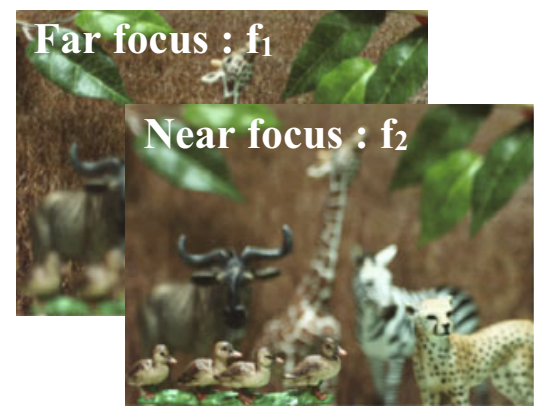

a. Captured images

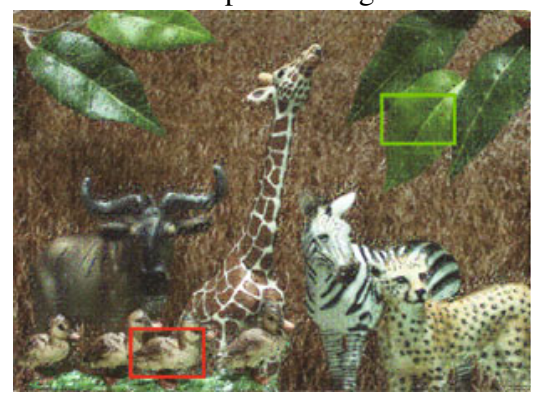

b. Recovered image

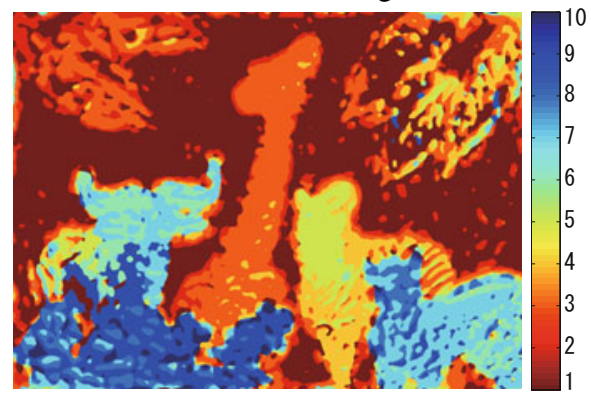

c. Depth map

Fig. 8. Conventional DFD
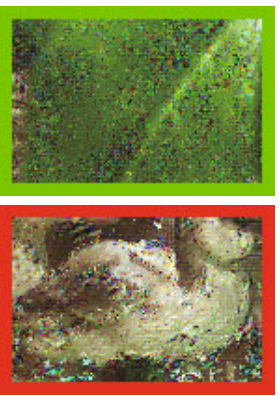

a. Conventional DFD

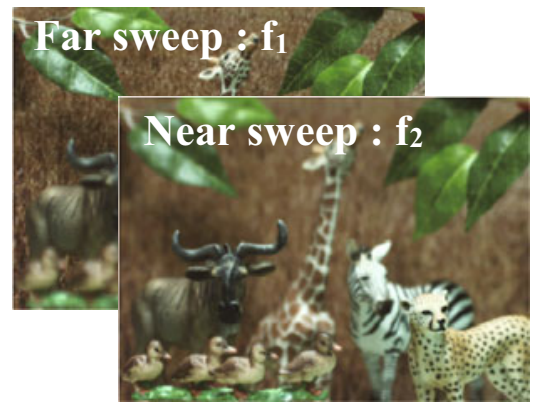

a. Captured images

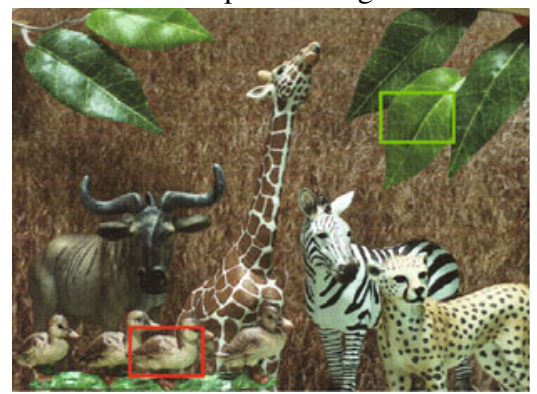

b. Recovered image

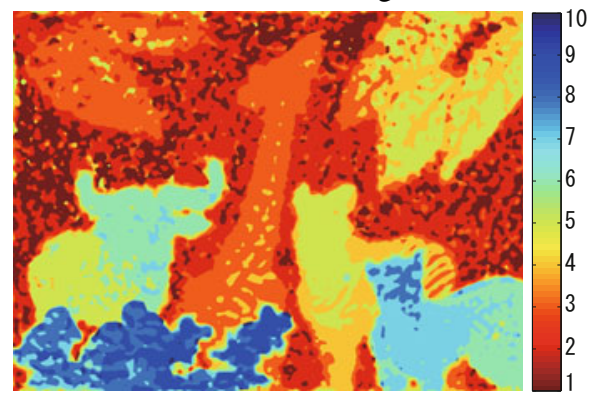

c. Depth map

Fig. 9. Half-sweep imaging

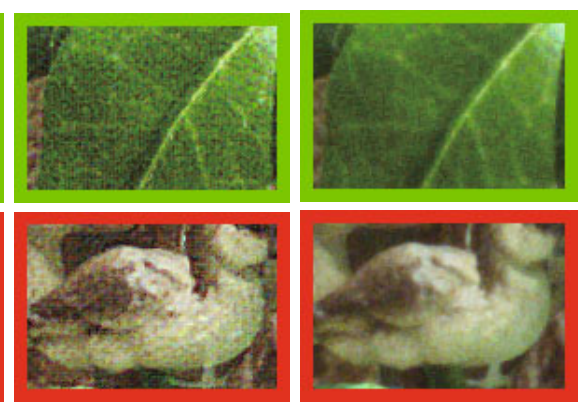

b. Half-sweep imaging DFD c. Ground truth $(f / \#=16)$

Fig. 10. Zoom up potion of images 


\section{References}

1. Dowski, E.R., Cathey, W.T.: Single-lens single-image incoherent passive-ranging systems. Applied Optics 33(29) (1994)

2. Green, P., Sun, W., Matusik, W., Durand, F.: Multiple-aperture photography. In: Proc. ACM SIGGRAPH (2007)

3. Hiura, S., Matsuyama, T.: Depth measurement by the multi-focus camera. In: Proc. IEEE Int. Conf. Computer Vision and Pattern Recognition, pp. 953-959 (1998)

4. Kuthirummal, S., Nagahara, H., Zhou, C., Nayar, S.K.: Flexible depth of field photography. IEEE Trans. Pattern Analysis and Machine Intelligence 33(1), 58-71 (2011)

5. Levin, A., Fergus, R., Durand, F., Freeman, W.T.: Image and depth from a conventional camera with a coded aperture. ACM Transactions on Graphics 26(3) (2007)

6. Levin, A., Hasinoff, S., Green, P., Durand, F., Freeman, W.T.: 4d frequency analysis of computational cameras for depth of field extension. ACM Transactions on Graphics (2009)

7. Nagahara, H., Kuthirummal, S., Zhou, C., Nayar, S.K.: Flexible Depth of Field Photography. In: Forsyth, D., Torr, P., Zisserman, A. (eds.) ECCV 2008, Part IV. LNCS, vol. 5305, pp. 60-73. Springer, Heidelberg (2008)

8. Nagahara, H., Zhou, C., Watanabe, T., Ishiguro, H., Nayar, S.K.: Programmable Aperture Camera Using 1CoS. In: Daniilidis, K., Maragos, P., Paragios, N. (eds.) ECCV 2010, Part VI. LNCS, vol. 6316, pp. 337-350. Springer, Heidelberg (2010)

9. Pentland, A.: A new sense for depth of field. IEEE Tans. Pattern Analysis and Machine Intelligence 9(4), 423-430 (1978)

10. Hasinoff, S.W., Kutulakos, K.N., Durand, F., Freeman, W.T.: Time-constrained photography. In: Proc. IEEE International Conference on Computer Vision (2009)

11. Subbarao, M., Gurumoorthy, N.: Depth recovery from blurred edges. In: Proc. IEEE Int. Conf. Computer Vision and Pattern Recognition, pp. 498-503 (1988)

12. Veeraraghavan, A., Raskar, R., Agrawal, A., Mohan, A., Tumblin, J.: Dappled photography: Mask enhanced cameras for heterodyned light fields and coded aperture refocusing. In: ACM SIGGRAPH (2007)

13. Zhou, C., Lin, S., Nayar, S.: Coded aperture pairs for depth from defocus. In: Proc. IEEE International Conference on Computer Vision (2010)

14. Zhou, C., Nayar, S.K.: What are good apertures for defocus deblurring? In: IEEE International Conference on Computational Photography (2009) 